\title{
Legislación Financiera y Tributaria de las Comunidades Autónomas (enero-mayo 1991)
}

\author{
Carmen Fernández \\ Profesora Titular de Derecho Financiero y Tributario \\ Universidad de las Islas Baleares.
}

\section{ANDALUCIA}

- Orden de 22 de enero de 1992, de la Consejería de Economía y Hacienda, sobre gestión y control de las operaciones de anticipos de Caja fija (BOJA n. ${ }^{\circ}$ 9, de 31 de enero de 1992).

- Orden de 3 de marzo de 1992, de la Consejería de Economía y Hacienda, sobre delegación de competencias en el Secretario General Técnico y en los titulares de los órganos directivos de la Consejería (BOJA n. ${ }^{\circ} 21$, de 10 de marzo de 1992).

\section{ARAGON}

- Orden de 10 de enero de 1992, del Departamento de Economía y Hacienda, por la que se desarrolla el Decreto 185/1991, de 5 de noviembre, regulador de la estructura orgánica del Departamento de Economía y Hacienda (BOA n. ${ }^{\circ} 8$, de 22 de enero de 1992).

- Orden de 14 de enero de 1992, del Departamento de Economía y Hacienda, por la que se determinan las condiciones a las que ha de ajustarse la prórroga del Presupuesto de la Comunidad Autónoma de Aragón para 1991 (BOA n..$^{\circ}$, de 22 de enero de 1992).

- Ley 5/1992, de 13 de abril, de las Cortes de Aragón, por la que se fijan las características básicas de las operaciones de endeudamiento autorizadas por la Ley 3/1991, de 10 de enero, de Presupuestos Generales de la Comunidad Autónoma de Aragón para 1991 (BOA n. ${ }^{\circ} 48$, de 27 de abril de 1992).

- Ley 6/1992, de 4 de mayo, de las Cortes de Aragón, de Presupuestos de la Comunidad Autónoma de Aragón para 1992 (BOA n. 51 suplemento, de 6 de mayo de 1992). 


\section{ASTURIAS}

- Resolución de 29 de enero de 1992, de la Consejería de Hacienda, Economía y Planificación, por la que se dictan instrucciones para la gestión de los ingresos figurados en el capítulo 3 del Presupuesto de Ingresos de la Administración del Principado de Asturias (BOPA n. ${ }^{\circ}$ 55, de 6 de marzo de 1992).

- Decreto 23/1992, de 6 de marzo, de la Consejería de Hacienda, Economía y Planificación, por el que se regula la formación y actualización del inventario de bienes muebles y la relación de bienes muebles no inventariables (BOPA n. ${ }^{\circ} 78$, de 2 de abril de 1992).

\section{BALEARES}

- Decreto 103/1991, de 5 de diciembre, del Gobierno Balear, por el que se regula el funcionamiento del «Sistema de Información de cuentas" (SICO) (BOCAIB n. ${ }^{\circ}$ 5, de 11 de enero de 1992).

- Decreto 1/1992, de 16 de enero, del Gobierno Balear, por el que se desarrolla el apartado 3 de la disposición adicional primera de la Ley 11/1991, de 13 de diciembre, de Presupuestos Generales de la Comunidad Autónoma de las Islas Baleares para el año 1992 y se modifica parcialmente el Decreto 102/1990, de 13 de diciembre, por el que se aprobó el Reglamento de la Ley 13/1990 (BOCAIB n. ${ }^{\circ} 8$, de 18 de enero de 1992).

- Orden de 16 de enero de 1992, de la Consellería de Economía y Hacienda, por la que se desarrolla la regulación sobre tributación de juegos de suerte, envite o azar y del Impuesto sobre el Juego del Bingo (BOCAIB n. ${ }^{\circ} 8$, de 18 de enero de 1992).

- Decreto 15/1992, de 27 de febrero, del Gobierno Balear, por el que se aprueba el Reglamento para el desarrollo del canon de saneamiento de aguas establecido por la Ley 9/1991, de 27 de noviembre (BOCAIB n. ${ }^{\circ}$ 34, de 19 de marzo de 1992).

- Orden de 18 de marzo de 1992, de la Consellería de Economía y Hacienda, por la que se desarrolla parcialmente el Decreto 15/1992, de 27 de febrero, que aprueba el Reglamento del canon de saneamiento de aguas residuales establecido por la Ley 9/1991, de 27 de noviembre (BOCAIB n. ${ }^{\circ} 38$, de 28 de marzo de 1992).

\section{CANARIAS}

- Orden de 27 de diciembre de 1991, por la que se establecen los requisitos que deben cumplir las garantías constituidas y a constituir 
para asegurar el pago aplazado de los Tributos de Importación gestionados y recaudados por la Comunidad Autónoma de Canarias como consecuencia de la entrada en vigor del Real Decreto-Ley 5/1991, de 20 de diciembre (BOCAC $n .^{\circ} 1$, de 1 de enero de 1992).

- Resolución de 9 de enero de 1992, de la Intervención General, por la que se dictan instrucciones provisionales para la Contabilidad del Presupuesto del ejercicio 1992 para los Organismos Autónomos (BOCAC n. ${ }^{\circ} 11$, de 24 de enero de 1992).

- Resolución de 28 de enero de 1992, de la Intervención General, por la que se establece el modelo de procedimiento e informe a utilizar en Auditorías Internas a los Centros de Servicios Sociales (BOCAC n. ${ }^{\circ} 24$, de 19 de febrero de 1992).

- Decreto 25/1992, de 28 de febrero, por el que se delega en los miembros del Gobierno el otorgamiento de subvenciones por razones de interés público (BOCAC n. ${ }^{\circ} 29$, de 2 de marzo de 1992).

- Orden de 28 de febrero de 1992, por la que se dictan normas sobre las incorporaciones de remanentes de crédito de los Presupuestos Generales de la Comunidad Autónoma de Canarias para el ejercicio de 1992 (BOCAC n. ${ }^{\circ} 32$, de 9 de marzo de 1992).

- Orden de 28 de febrero de 1992, sobre tramitación de expedientes de modificaciones de crédito (BOCAC n. ${ }^{\circ} 34$, de 13 de marzo de 1992).

- Decreto 30/1992, de 28 de febrero, por el que se encomienda a las Oficinas de Distrito Hipotecario, a cargo de Registradores de la Propiedad, la gestión y liquidación del Impuesto sobre Transmisiones Patrimoniales y Actos Jurídicos Documentados (BOCAC n. ${ }^{\circ} 39$, de 25 de marzo de 1992).

\section{CANTABRIA}

- Orden de 30 de octubre de 1991, de la Consejería de Economía, Hacienda y Presupuestos, sobre implantación en la contabilidad de los ingresos públicos del documento denominado "abonaré» de la Diputación Regional de Cantabria, y procedimiento para su gestión y control (BOC n. ${ }^{\circ}$, de 10 de enero de 1992).

- Decreto 5/1992, de 28 de enero, del Consejo de Gobierno, por el que se atribuyen competencias a las Oficinas Liquidadoras de Distrito Hipotecario a cargo de registradores de la Propiedad, para la gestión, 
liquidación y recaudación del Impuesto sobre Transmisiones Patrimoniales y Actos Jurídicos Documentados (BOC n. ${ }^{\circ} 29$, de 10 de febrero de 1992).

- Decreto 14/1992, de 31 de enero, sobre estructura orgánica de la Consejería de Economía, Hacienda y Presupuesto (BOC n. ${ }^{\circ} 3$, de 14 de febrero de 1992).

- Orden de 29 de enero de 1992, de la Consejería de Economía, Hacienda y Presupuesto, por la que se crea la Subcomisión para Fondos y Ayudas Comunitarias (BOC n. ${ }^{\circ}$ 36, de 19 de febrero de 1992).

- Ley $2 / 1992$, de 26 de febrero, por la que se establece el recargo provincial sobre las cuotas mínimas del Impuesto sobre Actividades Económicas (BOC n..$^{\circ}$, de 9 de marzo de 1992).

\section{CASTILLA-LA MANCHA}

- Decreto 13/1992, de 11 de febrero, sobre funciones de las Oficinas de Distrito Hipotecario para la gestión y liquidación del Impuesto sobre Transmisiones Patrimoniales y Actos Jurídicos Documentados (DOCM n. ${ }^{\circ} 15$, de 26 de febrero de 1992).

\section{CASTILLA Y LEON}

- Orden de 7 de enero de 1992, de la Consejería de Economía y Hacienda, por la que se aprueban nuevos modelos de efectos timbrados para la recaudación de tasas por prestación de determinados servicios de Inspección Técnica de Vehículos (BOCL n. ${ }^{\circ} 8$, de 14 de enero de 1992).

- Decreto 2/1992, de 16 de enero, del Gobierno de Castilla y León, por el que se aprueba el Reglamento de Organización y Funcionamiento del Consejo Económico y Social de Castilla y León (BOCL n. ${ }^{\circ} 13$, de 21 de enero de 1992).

- Orden de 7 de enero de 1992, de la Consejería de Economía y Hacienda, por la que se publican las tarifas actualizadas de las tasas propias de la Comunidad, en virtud de lo dispuesto en la Ley de Presupuestos Generales de la Comunidad para 1992 (BOCL n. ${ }^{\circ} 13$, de 21 de enero de 1992).

- Orden de 22 de enero de 1992, de la Consejería de Presidencia y Administración Territorial, por la que se establece el plazo para la 
CRONICAS

solicitud de declaración de territorios menos desarrollados a los efectos previstos en la Ley 7/1991, por la que se regula el Fondo de Compensación Regional (BOCL n. ${ }^{\circ}$ 17, de 27 de enero de 1992).

- Decreto 6/1992, de 23 de enero, sobre Estructura Orgánica de la Consejería de Economía y Hacienda (BOCL n. ${ }^{\circ}$ 17, de 27 de enero de 1992).

- Decreto 19/1992, de 6 de febrero, por el que se señala el plazo para que las Diputaciones provinciales cumplimenten los trámites establecidos en el artículo $4 .^{\circ}$ de la Ley 8/1989, de 9 de noviembre, de regulación transitoria del Fondo de Compensación Regional, para ejecutar el crédito correspondiente que figura en los Presupuestos de la Comunidad para 1992 (BOCL n. ${ }^{\circ} 29$, de 11 de febrero de 1992).

- Orden de 28 de enero de 1992, de la Consejería de Presidencia y Administración Territorial, por la que se amplía el plazo para la solicitud de declaración de territorios menos desarrollados a los efectos previstos en la Ley 7/1991, por la que se regula el Fondo de Compensación Regional (BOCL n. ${ }^{\circ} 44$, de 3 de marzo de 1992).

- Resolución de 18 de marzo de 1992, de la Dirección General de Tributos y Política Financiera, sobre instrucciones para el pago de los intereses de la Deuda Pública de la Comunidad de Castilla y León, Emisión 1986, Serie A (BOCL n. ${ }^{\circ} 61$, de 27 de marzo de 1992).

- Orden de 9 de abril de 1992, de la Consejería de Economía y Hacienda, por la que se desarrolla la Estructura Orgánica de los Servicios Centrales de la Consejería de Economía y Hacienda (BOCL n. ${ }^{\circ} 73$, de 14 de abril de 1992).

- Orden de 6 de mayo de 1992, de la Consejería de Economía y Hacienda, por la que se dictan normas para la elaboración de los Presupuestos Generales de la Comunidad de Castilla y León para 1993 (BOCL n. ${ }^{\circ}$ 94, de 19 de mayo de 1992).

- Resolución de 18 de mayo de 1992, de la Dirección General de Tributos y Política Financiera, sobre instrucciones para el pago de los intereses de la Deuda Pública de la Comunidad de Castilla y León, Emisión 1985 (BOCL n. ${ }^{\circ}$ 99, de 26 de mayo de 1992).

\section{CATALUÑa}

- Ley 33/1991, de 24 de diciembre, de Tasas y Precios Públicos de la Generalidad de Cataluña (DOGC n. ${ }^{\circ}$ 1546, de 24 de enero de 1992). 
- Resolución de 27 de enero de 1992, del Departamento de Economía y Finanzas, relativa a las instrucciones para el pago de los intereses correspondientes al vencimiento de 14 de febrero de 1992 y de la cuarta amortización parcial de la Emisión de Deuda Pública de la Generalidad de Cataluña de 14 de febrero de 1985 (DOGC n. ${ }^{\circ} 1552$, de 7 de febrero de 1992).

- Decreto 97/1992, de 28 de abril, por el que se acuerda la emisión de deuda pública de la Generalidad de Cataluña por un importe de 15.000 millones de pesetas, ampliable hasta 25.000 millones (DOGC $n .^{\circ} 1593$, de 13 de mayo de 1992).

- Resolución de 14 de mayo de 1992, relativa a las instrucciones para el pago de los intereses correspondientes al $5 .^{\circ}$ vencimiento de la emisión de Deuda Pública de la Generalidad de Cataluña (DOGC n. ${ }^{\circ} 1600$, de 29 de mayo de 1992).

- Resolución de 19 de mayo de 1992, relativa a las instrucciones para el pago de los intereses y la amortización correspondiente al $9 .^{\circ}$ vencimiento de intereses de la emisión de Deuda Pública de la Generalidad de Cataluña (DOGC n. ${ }^{\circ} 1600$, de 29 de mayo de 1992).

\section{EXTREMADURA}

- Decreto 2/1992, de 28 de enero, de la Consejería de Economía y Hacienda, sobre funciones de Gestión y Liquidación del Impuesto sobre Transmisiones Patrimoniales y Actos Jurídicos Documentados por las Oficinas de Distrito Hipotecario a cargo de Registradores de la Propiedad (DOE n. ${ }^{\circ} 11$, de 6 de febrero de 1992).

- Orden de 30 de enero de 1992, de la Consejería de Economía y Hacienda, por la que se publican los modelos a enviar por parte de las Consejerías y centros directivos de la Junta de Extremadura para iniciar la vía ejecutiva en las deudas no satisfechas en período voluntario (DOE n. ${ }^{\circ} 11$, de 6 de febrero de 1992).

- Orden de 20 de febrero de 1992, de la Consejería de Economía y Hacienda, por la que se establece la cuantía del Premio de Liquidación a los señores Registradores que dentro del ámbito de la Comunidad Autónoma, tengan a su cargo oficinas liquidadoras de distrito hipotecario (DOE n. ${ }^{\circ}$ 17, de 27 de febrero de 1992).

- Decreto 28/1992, de 24 de marzo, de la Junta de Extremadura, de la Unión Presupuestaria (DOE n. ${ }^{\circ} 25$, de 31 de marzo de 1992). 
CRONICAS

\section{GALICIA}

- Decreto 421/1991, de 30 de diciembre, por el que se desarrolla el artículo 22 de la Ley 15/1991, de 28 de diciembre, de presupuestos generales de la Comunidad Autónoma de Galicia para el ejercicio de 1992, referente a operaciones de tesorería (DOG n. ${ }^{\circ} 4$, de 8 de enero de 1992).

- Decreto 422/1991, de 30 de diciembre, por el que se encomienda a las Oficinas de Distrito Hipotecario funciones de gestión y liquidación en el impuesto sobre transmisiones patrimoniales y actos jurídicos documentados (DOG n. ${ }^{\circ} 4$, de 8 de enero de 1992).

- Orden de 23 de diciembre de 1991, por la que se aprueban normas relativas al pago de la tasa fiscal que recae sobre máquinas recreativas o aparatos automáticos aptos para juegos de azar (DOG n. ${ }^{\circ} 4$, de 8 de enero de 1992).

- Orden de 19 de diciembre de 1991, por la que se aprueba la instrucción provisional de contabilidad de los organismos autónomos de la Comunidad Autónoma de Galicia (DOG n. ${ }^{\circ} 21$, de 31 de enero de 1992).

- Orden de 20 de diciembre de 1991, por la que se dictan instrucciones para la apertura de la contabilidad de los organismos autónomos de la Comunidad Autónoma de Galicia (DOG n. ${ }^{\circ} 23$, de 4 de febrero de 1992).

- Decreto 16/1992, de 29 de enero, de cooperación con las entidades locales (DOG n. ${ }^{\circ} 24$, de 5 de febrero de 1992).

- Decreto 21/1992, de 29 de enero, por el que se desarrolla el artículo 31 de la Ley 15/1991, de 28 de diciembre, de presupuestos generales de la Comunidad Autónoma de Galicia para 1992 (DOG n. ${ }^{\circ} 25$, de 6 de febrero de 1992).

- Ley 2/1992, de 17 de marzo, de concesión de una paga al personal al servicio de la Administración de la Comunidad Autónoma, modificación del incremento retributivo previsto en la Ley 15/1991, de 28 de diciembre, y sobre concesión de un crédito extraordinario por importe de dos mil trescientos ochenta millones de pesetas $\mathbf{2} .380 .000 .000$ de pesetas), al vigente presupuesto de gastos, para hacer efectivas dichas medidas (DOG n. ${ }^{\circ}$ 56, de 23 de marzo de 1992).

\section{LA RIOJA}

- Orden (sin fecha), de la Consejería de Hacienda y Economía, por la que se encomienda a las oficinas liquidadoras de partido a cargo de los 
registradores de la propiedad, funciones en la gestión y liquidación de los Impuestos de Transmisiones Patrimoniales y Actos Jurídicos Documentados y de Sucesiones y Donaciones (BOR n. ${ }^{\circ} 42$, de 7 de abril de 1992).

- Ley 1/1992, de 7 de abril, de la Diputación General de La Rioja, de Presupuestos Generales de la Comunidad Autónoma de La Rioja para 1992 (BOR n. ${ }^{\circ} 43$, de 9 de abril de 1992).

- Decreto 12/1992, de 2 de abril, del Consejo de Gobierno, de normas reguladoras del procedimiento de concesión y gestión de subvenciones y ayudas de la Comunidad Autónoma de la Rioja (BOR n. ${ }^{\circ} 45$, de 14 de abril de 1992).

- Decreto 13/1992, de 2 de abril, del Consejo de Gobierno, por el que se regulan y desarrollan las previsiones contenidas en el artículo 95 de la Ley General Presupuestaria, en materia de fiscalización de gastos (BOR n. ${ }^{\circ} 45$, de 14 de abril de 1992).

- Orden de 20 de mayo de 1992, de la Consejería de Economía y Hacienda, por la que se dictan normas para la aplicación de la Ley 1/1992, de Presupuestos Generales de la Comunidad Autónoma para 1992, en relación con las retribuciones de los funcionarios (BOR n. ${ }^{\circ} 63$, de 26 de mayo de 1992).

\section{MADRID}

- Orden 5/1992, de 10 de enero, del Consejero de Hacienda, por la que se delegan determinadas funciones en el Director General de Presupuestos (BOM n. ${ }^{\circ} 13$, de 16 de enero de 1992).

- Orden 272/1992, de 7 de febrero, del Consejero de Hacienda, por la que se dispone la emisión de Deuda Pública interior por importe de 19.000 millones de pesetas (BOM n. ${ }^{\circ} 42$, de 19 de febrero de 1992).

- Orden 432/1992, de 6 de marzo, del Consejero de Hacienda, por la que se regula el procedimiento de las incorporaciones de crédito (BOM n. ${ }^{\circ} 58$, de 9 de marzo de 1992).

- Orden 527/1992, de 3 de marzo, del Consejero de Hacienda, por la que se desarrollan las estructuras orgánicas de las Intervenciones Delegadas en las Consejerías de Transportes, Política Territorial y Educación y Cultura, y se modifica parcialmente la de la Consejería de Integración Social (BOM n. ${ }^{\circ} 68$, de 20 de marzo de 1992). 
CRONICAS

- Ley 1/1992, de 12 de marzo, de la Asamblea de Madrid, de Tasas y Precios Públicos de la Comunidad de Madrid (BOM n. ${ }^{\circ} 77$, de 31 de marzo de 1992. Corrección de errores BOM n. ${ }^{\circ} 84$, de 8 de abril de 1992).

- Ley 2/1992, de 30 de abril, de la Asamblea de Madrid, de Presupuestos Generales de la Comunidad de Madrid para 1992 (BOM n. ${ }^{\circ}$ 109, de 8 de mayo de 1992).

- Acuerdo de 7 de mayo de 1992, del Consejo de Gobierno, por el que se delegan determinadas facultades en los excelentísimos señores Consejeros (BOM n. ${ }^{\circ} 114$, de 14 de mayo de 1992).

- Orden 1212/1992, de 12 de mayo, del Consejero de Hacienda, por la que se dictan las normas de elaboración de los Presupuestos Generales de la Comunidad de Madrid para 1993 (BOM n. ${ }^{\circ} 117$, de 18 de mayo de 1992).

- Acuerdo del Consejo de Gobierno, de 22 de mayo de 1992, por el que se establece el catálogo de bienes, servicios y actividades susceptibles de ser retribuídos mediante precio público, en el ámbito de la Comunidad de Madrid (BOM n. ${ }^{\circ} 125$, de 27 de mayo de 1992).

\section{MURCIA}

- Decreto 3/1992, de 17 de enero, del Consejo de Gobierno, por el que se crea la Consejería de Economía, Hacienda y Fomento (BORM n. ${ }^{\circ} 14$, de 18 de enero de 1992).

- Orden de 21 de enero de 1992, de la Consejería de Economía, Hacienda y Fomento, por la que se desarrolla lo dispuesto en el artículo 20 de la Ley 3/1991, de 23 de diciembre de Presupuestos Generales de la Región de Murcia para 1992, actualizando las tarifas de las tasas gestionadas por la Administración Regional (BORM n. ${ }^{\circ} 23$, de 29 de enero de 1992).

- Decreto 7/1992, de 30 de enero, del Consejo de Gobierno, por el que se establecen los órganos directivos de la Consejería de Economía, Hacienda y Fomento (BORM n. ${ }^{\circ} 29$, de 5 de febrero de 1992).

- Decreto 2/1992, de 16 de enero, de la Consejería de Hacienda, por el que se encomiendan las funciones de gestión y liquidación del Impuesto sobre Transmisiones Patrimoniales y Actos Jurídicos Documentados a las oficinas Liquidadoras de Distrito Hipotecario de los Registradores de la Propiedad (BORM n. ${ }^{\circ} 41$, de 19 de febrero de 1992). 
- Orden de 11 de febrero de 1992, de la Consejería de Economía, Hacienda y Fomento, de delegación de determinadas competencias del titular del departamento en el Secretario General (BORM n. ${ }^{\circ} 42$, de 20 de febrero de 1992).

- Orden de 6 de abril de 1992, de la Consejería de Economía, Hacienda y Fomento, por la que se regulan determinadas normas sobre depuración de valores correspondientes a tributos cedidos, impuestos propios, tasas, multas y sanciones, y demás derechos económicos de la Hacienda Regional (BORM, n. ${ }^{\circ}$ 93, de 22 de abril de 1992).

\section{NAVARRA}

- Orden Foral 1223/1991, de 9 de diciembre, del Consejero de Economía y Hacienda, por la que se reestructuran, establecen y suprimen diversos negociados del Departamento (BON n. ${ }^{\circ} 4$, de 8 de enero de 1992).

- Decreto Foral 563/1991, de 23 de diciembre, del Gobierno de Navarra, por el que se amplía el ámbito de aplicación de la coordinación del Registro Fiscal de la Riqueza Territorial de Navarra con el Registro de la Propiedad, en los términos dispuestos en el Decreto Foral 54/1991, de 7 de febrero (BON n. ${ }^{\circ}$ 6, de 13 de enero de 1992).

- Decreto Foral 3/1992, de 7 de enero, del Gobierno de Navarra, por el que se modifica la tabla de retenciones a cuenta del Impuesto sobre la Renta de las Personas Físicas contenida en el artículo 135 del Reglamento del citado Impuesto (BON n. ${ }^{\circ}$ 7, de 15 de enero de 1992).

- Decreto 571/1991, de 30 de diciembre, del Gobierno de Navarra, por el que se da nueva redacción al número 2 del artículo 68 del Reglamento del Impuesto sobre Transmisiones Patrimoniales y Actos Jurídicos Documentados, sobre las condiciones del canje de los efectos timbrados (BON n. ${ }^{\circ}$ 8, de 17 de enero de 1992).

- Orden Foral 1319/1991, de 30 de diciembre, del Consejero de Economía y Hacienda, por la que se aprueban los modelos de impresos para la declaración-liquidación y pago de la Tasa Fiscal sobre el Juego correspondiente a máquinas o aparatos automáticos (BON n. ${ }^{\circ} 10$, de 22 de enero de 1992).

- Orden Foral 6/1992, de 13 de enero, del Consejero de Economía y Hacienda, por la que se crea el Negociado de Contabilidad de Deudores (BON n. ${ }^{\circ}$ 13, de 29 de enero de 1992). 
CRONICAS

- Acuerdo del Parlamento de Navarra, de 5 de febrero de 1992, por el que se aprueba el Decreto Foral 568/1991, de 23 de diciembre, de modificación parcial de la Ley Foral 24/1985, de 11 de diciembre, del Impuesto sobre el Valor Añadido (BON n. ${ }^{\circ} 19$, de 12 de febrero de 1992).

- Orden Foral 60/1992, de 28 de enero, del Consejero de Economía y Hacienda, por la que se aprueban las tablas de precios medios de venta de vehículos usados, de aplicación en los Impuestos sobre Sucesiones, Transmisiones Patrimoniales y Actos Jurídicos Documentados (BON n..$^{\circ} 20$, de 14 de febrero de 1992).

- Orden Foral 79/1992, de 7 de febrero, del Consejero de Economía y Hacienda, por la que se modifica la denominación del Negociado de Documentación, Información y Banco de Datos, así como las funciones asignadas al mismo (BON n. ${ }^{\circ} 21$, de 17 de febrero de 1992).

- Decreto Foral 54/1992, de 17 de febrero, del Gobierno de Navarra, por el que se dictan normas para la presentación de las declaraciones por los Impuestos sobre la Renta de las Personas Físicas y Extraordinario sobre el Patrimonio correspondientes al ejercicio de 1991 (BON n. ${ }^{\circ}$ 24, de 24 de febrero de 1992).

- Decreto Foral 55/1992, de 17 de febrero, del Gobierno de Navarra, por el que se modifican diversos artículos del Reglamento del Impuesto sobre el Valor Añadido referentes al régimen especial de recargo de equivalencia (BON n. ${ }^{\circ} 24$, de 24 de febrero de 1992).

- Orden Foral 118/1992, de 19 de febrero, del Consejero de Economía y Hacienda, por la que se crea en la Sección de Impuestos Indirectos el Negociado de Administración y Control (BON n. ${ }^{\circ} 29$, de 6 de marzo de 1992).

- Decreto Foral 86/1992, de 9 de marzo, por el que se modifica parcialmente la Ley Foral de 11 de diciembre de 1985, del Impuesto sobre el Valor Añadido (BON n. ${ }^{\circ}$ 35, de 20 de marzo de 1992).

- Orden Foral 147/1992, de 3 de marzo, del Consejero de Economía y Hacienda, por la que se aprueba el modelo de declaración del Impuesto sobre Sociedades correspondiente al ejercicio de 1991 (BON n. ${ }^{\circ} 37$, de 25 de marzo de 1992).

- Orden Foral 206/1992, de 23 de marzo, del Consejero de Economía y Hacienda, por la que se aprueban los modelos de declaración correspondientes a los Impuestos sobre la Renta de las Personas Físicas y 
Extraordinario sobre el Patrimonio del ejercicio de 1991 (BON n. ${ }^{\circ} 44$, de 10 de abril de 1992).

- Ley Foral 3/1992, de 14 de abril, del Parlamento de Navarra, de Cuentas Generales sobre la Renta de las Personas Físicas (BON n. ${ }^{\circ} 63$, de 35 de mayo de 1992).

- Ley Foral 7/1992, de 14 de mayo, por la que se autoriza al Gobierno de Navarra a prestar un aval de la Comunidad Foral de Navarra en favor de la Compañía Solano, S.A. (BON n..$^{\circ} 63$, de 25 de mayo de 1992).

\section{PAIS VASCO}

- Orden de 3 de diciembre de 1991, del Consejero de Hacienda y Finanzas, por la que se aprueban los modelos básicos de liquidación de tasas y justificantes de pago de precios públicos de la Administración de la Comunidad Autónoma del País Vasco (BOPV n. ${ }^{\circ} 14$, de 22 de enero de 1992).

- Orden de 30 de diciembre de 1992, del Consejero de Hacienda y Finanzas, por la que se establece la información económico-financiera que los Entes Públicos de Derecho Privado y Sociedades Públicas deben remitir al Departamento de Hacienda y Finanzas (BOPV n. ${ }^{\circ} 22$, de 3 de febrero de 1992).

- Decreto 698/1991, de 17 de diciembre, por el que se regula el régimen general de garantías y reintegros de las subvenciones con cargo a los Presupuestos Generales de la Comunidad Autónoma de Euskadi y se establecen los requisitos, régimen y obligaciones de las Entidades Colaboradoras que participan en su gestión (BOPV n. ${ }^{\circ} 26$, de 7 de febrero de 1992).

- Ley 1/1992, de 28 de enero, del Parlamento Vasco, por la que se aprueban los Presupuestos Generales de la Comunidad Autónoma de Euskadi para 1992 (BOPV n. ${ }^{\circ}$ 30, de 13 de febrero de 1992).

- Decreto 82/1992, de 7 de abril, por el que se establece el contenido de la documentación general necesaria para la creación de Sociedades Públicas pertenecientes a la Administración Institucional de la Comunidad Autónoma de Euskadi (BOPV n. ${ }^{\circ} 87$, de 11 de mayo de 1992).

- Decreto 110/1992, de 5 de mayo, por el que se dispone la emisión de Deuda Pública de Euskadi, interior y amortizable, formalizaba en 
obligaciones, por importe de trece mil quinientos millones (13.500.000.000) de pesetas (BOPV n. ${ }^{\circ} 87$, de 11 de mayo de 1992).

\section{COMUNIDAD VALENCIANA}

- Decreto 249/1991, de 23 de diciembre, del Consell de la Generalitat Valenciana, por el que se atribuyen determinadas competencias para la gestión y liquidación del Impuesto sobre Transmisiones Patrimoniales y Actos Jurídicos Documentados a las oficinas liquidadoras de distrito hipotecario (DOGV n. ${ }^{\circ} 1695$, de 2 de enero de 1992).

- Orden de 17 de diciembre de 1991, del Conseller de Economía y Hacienda, por la que se establece el procedimiento de ingreso de los tributos cedidos que se realicen a través de los distintos hipotecarios de la Comunidad Valenciana (DOGV n. ${ }^{\circ} 1698$, de 8 de enero de 1992).

- Decreto 8/1992, de 7 de enero, del Gobierno Valenciano, por el que se modifica y complementa el programa de pagarés de la Generalitat Valenciana (DOGV n. ${ }^{\circ} 1704$, de 16 de enero de 1992).

- Orden de 27 de enero de 1992, del Conseller de Economía y Hacienda, por la que se regula el procedimiento de gestión y modificaciones del presupuesto de gastos de la Generalitat Valenciana para 1992 (DOGV n. ${ }^{\circ} 1717$, de 5 de febrero de 1992).

- Decreto 15/1992, de 3 de febrero, del Gobierno Valenciano, por el que se desarrolla el artículo 32 de la Ley 7/1991, de 28 de diciembre, de Presupuestos de la Generalitat Valenciana para 1992 (DOGV n. ${ }^{\circ} 1720$, de 10 de febrero de 1992).

- Orden de 29 de enero de 1992, de la Consellería de Economía y Hacienda, por la que se regula la concesión de aplazamientos y fraccionamientos de deudas tributarias de los tributos cedidos y propios de la Generalitat Valenciana (DOGV n. ${ }^{\circ} 1728$, de 20 de febrero de 1992).

- Orden de 12 de febrero de 1992, del Conseller de Economía y Hacienda, por la que se establecen las cuantías de los precios públicos a percibir por la Consellería de Economía y Hacienda (DOGV n. ${ }^{\circ} 1732$, de 26 de febrero de 1992).

- Orden de 20 de febrero de 1992, del Conseller de Economía y Hacienda, por la que se desarrolla el Decreto 15/1992 en relación con el artículo 32 de la Ley de Presupuestos para 1992 (DOGV n. ${ }^{\circ} 1737$, de 4 de marzo de 1992). 
- Ley 2/1992, de 26 de marzo, de las Cortes Valencianas, de saneamiento de las aguas residuales de la Comunidad Valenciana (DOGV n. $^{\circ}$ 1761, de 8 de abril de 1992).

- Decreto 54/1992, de 30 de marzo, del Gobierno Valenciano, por el que se aprueba la ampliación del límite del endeudamiento de la Generalitat Valenciana para el ejercicio 1992 en la cuantía del endeudamiento no utilizado en el ejercicio 1991 (DOGV n. ${ }^{\circ} 1762$, de 9 de abril de 1992).

- Orden de 13 de abril de 1992, de la Consellería de Economía y Hacienda, por la que se amplía la gradación de emisiones de obligaciones de la Generalitat Valenciana correspondientes al Programa Financiero 1991 (DOGV n. ${ }^{\circ} 1783$, de 15 de mayo de 1992).

\section{ABREVIATURAS UTILIZADAS}

BOA Boletín Oficial de Aragón

BOC Boletín Oficial de Cantabria

BOCAC Boletín Oficial de la Comunidad Autónoma de Canarias

BOCAIB Boletín Oficial de la Comunidad Autónoma de las Islas Baleares

BOCL Boletín Oficial de Castilla y León

BOJA Boletín Oficial de la Junta de Andalucía

BOM Boletín Oficial de Madrid

BON Boletín Oficial de Navarra

BOPA Boletín Oficial del Principado de Asturias

BOPV Boletín Oficial del País Vasco

BOR Boletín Oficial de la Rioja

BORM Boletín Oficial de la Región de Murcia

DOCM Diario Oficial de Castilla-La Mancha

DOE Diario Oficial de Extremadura

DOG Diario Oficial de Galicia

DOGC Diario Oficial de la Generalidad de Cataluña

DOGV Diario Oficial de la Generalidad Valenciana 


\section{Sección Jurisprudencia}


REAL-1992, núm. 255-256. FERNÁNDEZ, CARMEN. LEGISLACIÓN FINANCIERA Y TRIBUTARIA ...

REAL-1992, núm. 255-256. FERNÁNDEZ, CARMEN. LEGISLACIÓN FINANCIERA Y TRIBUTARIA ... 